\title{
Ergonomics in the operating room
}

\author{
Jenny Gremark Simonsen*, Inger Arvidsson and Catarina Nordander \\ Department of Occupational and Environmental Medicine, University Hospital, SE-221 85 Lund, Sweden
}

\begin{abstract}
Surgical staff is considered to have several ergonomic risk factors, but their physical workload and musculoskeletal health have seldom been evaluated. Clinical examinations of neck and upper extremities were performed in 99 theatre nurses and 93 assisting nurses, all females. Their physical workload was assessed by questionnaire, and by technical measurements of postures, movements and muscular load in subgroups of both categories.

The prevalences of diagnoses in neck/shoulders were not remarkably high in the nurses, compared to other occupational groups. In elbows/hands though, the prevalence was rather high among assistant nurses, 13 vs. $5 \%$ in theatre nurses; POR 3.0 (CI $95 \% 1.0-8.9$ ).

Theatre nurses experienced prolonged static postures in the questionnaire, whilst assistant nurses reported high physical load. Accordingly, the technical measurements showed more strenuous working postures in the theatre nurses, but lower wrist angular velocities. The time proportion of muscular rest in m.trapezius was rather high in both groups.

Although both groups had some stressful loads they had relatively low prevalence of diagnoses in neck/shoulders. The high proportion of muscular rest may be protective. The elevated risk of elbows/hands diagnoses among assistant nurses may be explained by strenuous work tasks of short duration.
\end{abstract}

Keywords: Physical load, technical measurements, mechanical and physical indices, musculoskeletal disorders

\section{Introduction}

Operation staff is considered to have several known risk factors for musculoskeletal disorders, such as long term static postures and heavy loads. Their physical workload and musculoskeletal health have however seldom been evaluated $[2,6]$. Female theatre nurses and assistant nurses in four medium sized hospitals were investigated. The survey is a part of a larger prospective study. The aim was to quantify disorder prevalence and to measure the physical workload among surgical staff.

\footnotetext{
*Corresponding author. e-mail:jenny.gremarksimonsen@med.lu.se
}

\section{Methods}

\subsection{Methods}

The study groups consisted of 99 female theatre nurses and 93 assistant nurses.

Each subject was examined clinically according to a standardized method in the neck/shoulders and elbows/hands, which have previously been used in many different occupations [5]. Findings were registered and if they met certain predefined criteria, diagnoses were set. The proportions of participants with at least one diagnosis in the neck/shoulders and elbows/hands respectively, were calculated.

The nurses answered a questionnaire about the physical workload in a 3-point scale. Postures and repetitive movements were classified into a mechanical index, and physical loads and heavy lifting into a physical index [1]. Based on the sum of the score, the 
participants were subdivided into four exposure categories for each index.

Finally, physical load was recorded by technical measurements on sub-groups, consisting of 12 participants in each occupation. Inclinometry was used for upper arm elevation and head forward bending [3], goniometry for movement velocities in the wrist [4] and bipolar surface electromyography (EMG) was used for recording of the muscular activity of the trapezius muscle. The proportion of muscular rest, i.e. an amplitude level less than $0.5 \%$ of maximal voluntary contraction, was calculated [4].

Each measurement covered an entire working day with all the occurring tasks. The same methods have earlier been used in many different occupations, enabling comparisons $[3,4]$.

\section{Results}

\subsection{Musculoskeletal disorders}

The prevalences of diagnoses in neck/shoulders were similar for the groups: $25 \%$ of the assistant nurses vs. $17 \%$ of the theatre nurses and had at least one diagnosis; prevalence odds ratio (POR) 1.6 (95\% CI 0.8 - 3.3; adjusted for age). This was not remarkably high as compared to other workgroups, examined by the same methods. On the other hand assistant nurses had a rather high prevalence of diagnoses in elbows/hands. As many as $13 \%$ had at least one diagnosis compared to $5 \%$ among the theatre nurses POR 3,0 (95\% CI 1.0 - 1.9; adjusted for age). The prevalence of diagnoses in elbows/hands in the assistant nurses was almost as high as among cleaners (Figure 1).

\subsection{Physical workload}

Concerning the mechanical index, $68 \%$ of the theatre nurses reported awkward postures and repetitive movements in the highest exposure category, compared to $52 \%$ of the assistant nurses. Contrary for the physical index, $44 \%$ of the theatre nurses and $61 \%$ of the assistant nurses reported high physical loads in the highest exposure category.

The technical measurements showed median right upper arm elevation (group mean) of $29^{\circ}$ (SD 4.6) for theatre nurses and $25^{\circ}$ (SD 3.9 ) for assistant nurses $\left(50^{\text {th }}\right.$ percentile; $\left.\mathrm{p}<0.05\right)$. Corresponding values for head forward bending were $22^{\circ}$ (SD 7.0) and $12^{\circ}(\mathrm{SD} \mathrm{5.6}) ; \mathrm{p}<0.01$. Compared to other work groups, where exposure was assessed by identical methods, the theatre nurses' exposure levels were similar to hairdressers' for the upper arm and to poultry processing workers' for the head. For movement velocities in the right wrist, assistant nurses showed higher values than the theatre nurses $(9.7 \%$ s (SD 3.9) vs. $6.9 \%$ s (SD 3.0); $<<0.05$, but both relatively low compared to other professions.

The proportion of muscular rest in the trapezius muscle was rather high in both groups (theatre nurses $18 \%$ and assisting nurses $10 \%$ of the time). 


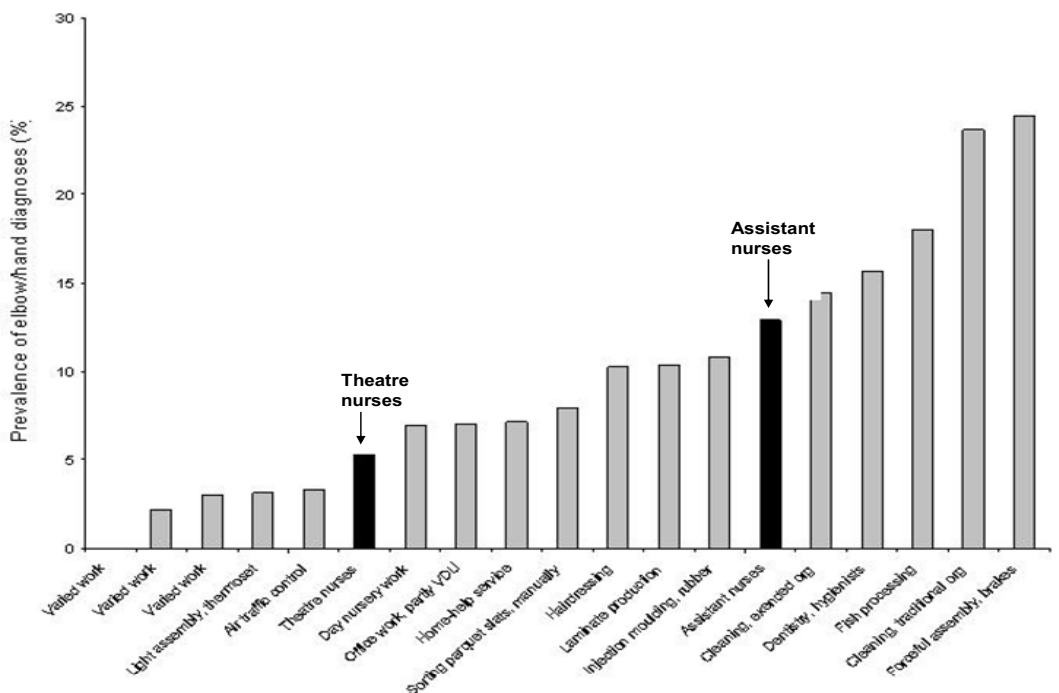

Figure 1. Prevalence of elbows/hands diagnoses among workers in 19 different occupations examined by the samr standardized clinical examination [5]. Surgical nurses and assistant nurses in black bars.

\section{Discussion}

The theatre nurses experienced prolonged and static postures while holding hooks, often with raised arms. Both groups reported transfer of patients as stressful work situations. However, although both theatre nurses and assistant nurses had stressful postures and loads, they had relatively low prevalence of diagnoses in neck/shoulders. This may be explained by the fact that they had a rather high proportion of muscular rest in the trapezius muscle, and thus time for recovery between strenuous work tasks.

Concerning elbows/hands, the assistant nurses showed high prevalence of diagnoses as compared to other groups. The technical measurements, which gave average values for the whole day, showed moderate exposure of the workload in the elbows/hands regions. However, assistant nurses reported high physical loads in the questionnaire and we suspect that strenuous work tasks of short duration explain the elevated risk. Examples of such tasks are adjusting handles of the operation table accessories and heavy lifting of instruments.

\section{References}

[1] I. Balogh, L. Eng, P. Ørbæk, J. Winkel., C. Nordander, K.Ohlsson,, J. Ektor-Andersen. and Malmö ShoulderNeck Study Group. Questionnaire-based mechanical exposure indices for large population studies - reliability, internal consistency and predicitive validity. Scand. J Work Environ Health. 27 (2001), 41-8.

[2] E. Bos, B. Krol, L. van der Star, J. Groothoff. Risk factors and musculoskeletal complaints in non-specialized nurses, IC nurses, operation room nurses, and X-ray technologists. Int Arch Occup Environ Health. 80 (2007), 198-206

[3] G.-Å. Hansson, I. Balogh, K. Ohlsson, L. Granqvist, C. Nordander, I. Arvidsson, et al. Physical workload in various types of work: Part I Wrist and forearm. Int. J. Ind. Ergon. 39 (2009), 221-331.

[4] G.-Å. Hansson, I. Balogh, K. Ohlsson, L. Granqvist, C. Nordander, I. Arvidsson, I. Åkesson, J. Unge, R. Rittner, U. Strömberg, S. Skerfving. Physical workload in various types of work: Part II. Neck, shoulder and upper arm. Int. J. Ind. Ergon. 40 (2010), 267-281.

[5] C. Nordander, K. Ohlsson, I. Åkesson, I. Arvidsson, I. Balogh, G.-Å. Hansson et al. Risk of musculoskeletal disorders among females and males in repetetive /constrained work. Ergonomics. 10 (2009), 1226-39.

[6] A. Sheikhzadeh, C. Gore C, J.D. Zuckerman, M. Nordin, Perioperating nurses and technicians' perceptions of ergonomic risk factors in the surgical environment. Appl Ergon. 40 (2009), 833-839. 